\title{
Straightforward Production of Bright, Polarization-Tunable Attosecond High-Harmonic Waveforms via Circularly Polarized High Harmonic Generation
}

\author{
Kevin M. Dorney ${ }^{1}$, Tingting Fan ${ }^{1}$, Jennifer L. Ellis ${ }^{1}$, Daniel D. Hickstein ${ }^{1}$, Christopher Mancuso ${ }^{1}$, Nathan \\ Brooks $^{1}$, Dmitriy Zusin ${ }^{1}$, Christian Gentry ${ }^{1}$, Patrik Grychtol ${ }^{1}$, Ronny Knut ${ }^{1}$, Tenio Popmintehev ${ }^{1}$, \\ Carlos Hernández-García ${ }^{2}$, Dejan B. Miloševici ${ }^{3,4,5}$, Henry C. Kapteyn ${ }^{1}$, Margaret M. Murnane ${ }^{1}$ \\ ${ }^{I}$ JILA - Department of Physics, University of Colorado Boulder and NIST, Boulder, Colorado, 80309 \\ ${ }^{2}$ Grupo de Investigación en Aplicaciones del Láser y Fotónica, Departamento de Física Aplicada, \\ University of Salamanca, E-37008 Salamanca, Spain \\ ${ }^{3}$ Faculty of Science, University of Sarajevo, 71000 Sarajevo, Bosnia and Herzegovina \\ ${ }^{4}$ Academy of Sciences and Arts of Bosnia and Herzegovina, 71000 Sarajevo, Bosnia and Herzegovina \\ ${ }^{5}$ Max-Born-Institut, 12489 Berlin, Germany \\ kevin.dorney@colorado.edu
}

\begin{abstract}
We experimentally demonstrate straightforward methodologies for generating high harmonics of arbitrary polarization state. Polarization control is realized by adjusting the intensity ratio of the bicircular driving field or by exploiting chirally dependent Cooper minima transitions. (C) 2018 The Author(s) OCIS codes: (140.7240) UV, EUV, and X-ray lasers; (020.2649) Strong field laser physics; (320.7110) Ultrafast nonlinear optics
\end{abstract}

The recent re-discovery of circularly polarized high harmonic generation (CPHHG) has resulted in a renaissance of high-energy, polarization-sculpted attosecond light sources, which are capable of interrogating ultrafast, elementspecific chiral dynamics in condensed matter and molecular systems [1]. In particular, CPHHG driven by bichromatic, counter-rotating laser fields - the bicircular field-has been at the forefront of the attosecond polarization resurgence due to the possibility of controlling the high-harmonic spectral, temporal, and polarization properties afforded via the manipulation of these unique two-color fields [1,2]. Practically, direct control over the spectrotemporal structure of the CPHHG emission process is attractive for generating bright, tailor-made highharmonic beamlets for ultrafast magneto-optical chiral spectroscopies and also isolated attosecond pulses of nearly arbitrary polarization. As such, numerous strategies have been proposed to achieve this level of control by exploiting the helicity-dependent aspects of the microscopic and macroscopic response to the external field [1]. Despite sincere efforts, the complexity of many methodologies has limited active polarization control in CPHHG to just a few experimental demonstrations [2,3].

In this work, we present two straightforward, yet distinct, methodologies for controlling the spectral distribution of left- and right-circularly polarized (LCP/RCP) harmonics in bicircular-driven CPHHG (Fig. 1A), thus allowing for active manipulation of the polarization of the underlying attosecond pulse trains (APTs). First, we find that the intensity ratio of a commensurate bicircular field - where $2 \omega_{1}=\omega_{2}$ - serves as a practical, real-time knob for smoothly varying the ellipticity of the APTs, independent of the CPHHG photon energy and bandwidth (Fig. 1B). Second, we show that a non-commensurate near- and short-wave IR (SWIR) field, combined with an effectively phase-matched geometry, can be utilized to extend the CPHHG cut-off to beyond the Cooper minimum in Ar [4], resulting in the natural production of bright, single-helicity harmonic spectra (and thus circularly polarized APTs) spanning the $M$ absorption edges of several magnetic materials (Fig. 1C).

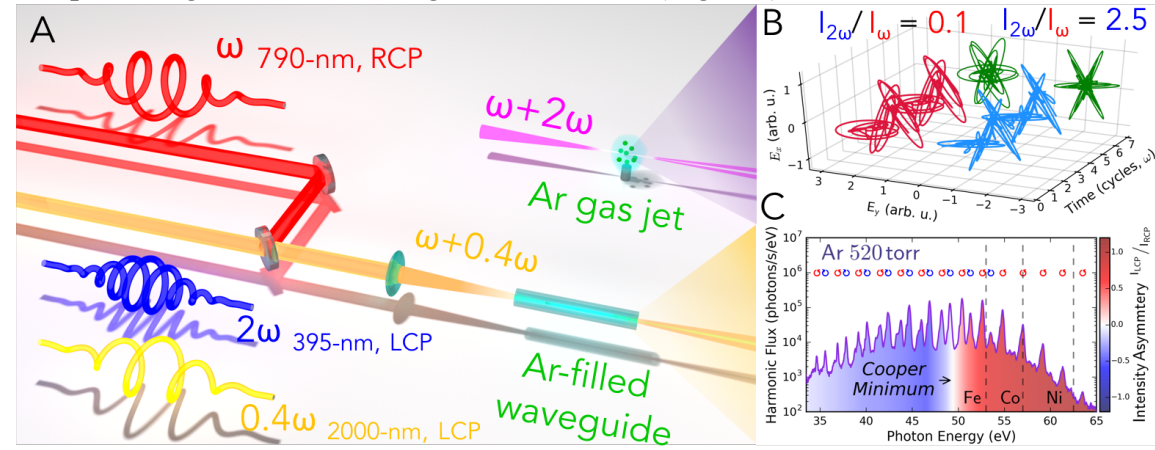

Figure 1. (A) Experimental scheme for bicircular-driven circularly polarized high harmonic generation (CPHHG). (B) Theoretical attosecond pulse trains obtained from highly chiral experimental CPHHG spectra generated in an Ar gas jet. (C) Short-wave-IR-driven CPHHG in a phase- 
matched waveguide geometry yields a bright spectrum that extends beyond the Cooper minimum in Ar, which generates a single-helicity spectrum covering prominent magneto-optical absorption edges.

In the experiment (Fig. 1A), circularly polarized high harmonics are generated via collinear mixing of the

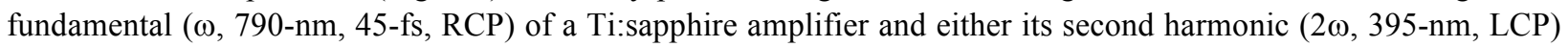
or a SWIR field $(0.4 \omega, 2000-\mathrm{nm}, \mathrm{LCP})$. The combined fields are then focused onto a supersonic gas jet $(\omega-2 \omega)$ or coupled into a hollow-core waveguide $(\omega-0.4 \omega)$. In either geometry, bright, circularly polarized high harmonic spectra are produced, which consist of a series of high-harmonic doublets possessing opposite helicities. The harmonics themselves exhibit a high degree of circularity, as indicated by the suppression of spin-forbidden harmonic orders between the circularly polarized doublets (Fig. 1C and Fig. 2A).

For commensurate, $\omega-2 \omega$, driven CPHHG in an Ar gas jet, the lower (higher) frequency harmonic in each doublet rotates with the $\omega(2 \omega)$ field, and these individual harmonics can be viewed as being produced by absorption of an additional $\omega(2 \omega)$ photon from the driving field. The intensity of the RCP (LCP) harmonics in the far-field are directly coupled to the photon density of each color in the two-color driving laser [2]. As such, we can actively control the intensity of either RCP or LCP high-harmonics in the far-field, thus generating highly chiral CPHHG spectra (Fig. 2A). In the time domain, controlling the chirality of the CPHHG process manifests as direct control over the ellipticity of the underlying APTs (Fig. 2B). As the intensity ratio is varied, the $x$ and $y$ components of the bicircular field that correspond to bright CPHHG emission also vary, which imparts ellipticity onto the APT. We confirm the dependence of this attosecond ellipticity on the CPHHG spectral chirality via macroscopic numerical simulations [5] of the upconversion process (Fig 2A, B). Most importantly, this attosecond ellipticity is uncoupled from the polarizations of the driving field, which allows for highly elliptical APTs to be produced without sacrificing the circular polarization of the emitted harmonics [2].
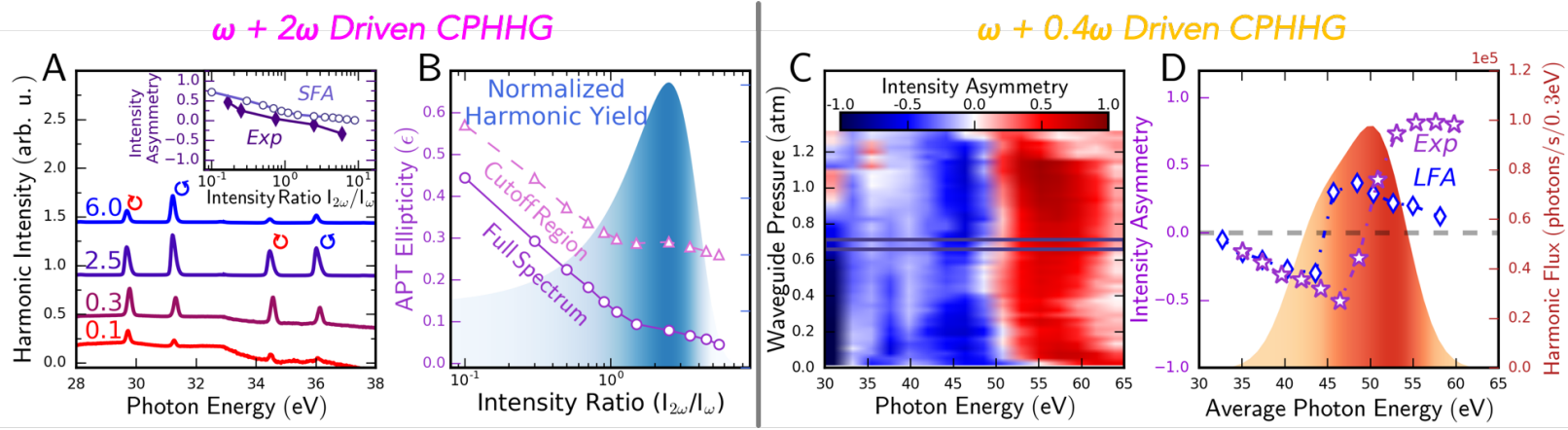

Fig 2. Controlling the ellipticity of APTs in CPHHG. (A) Experimental, $\omega-2 \omega$ CPHHG spectra in Ar generated at different intensity ratios, $I_{2 \omega} / I_{\omega}$, of the driving field (exact ratios indicated next to spectra). Inset depicts the intensity asymmetry, $\left(I_{R C P}-I_{L C P}\right) /\left(I_{R C P}+I_{L C P}\right)$, of the CPHHG spectra at different $I_{2 \omega} / I_{\omega}$. (B) Ellipticity of the APTs computed in Ar for experimental intensity ratios. (C) Experimental intensity asymmetries in $\omega-0.4 \omega$ driven CPHHG as a function of pressure in the hollow-core waveguide. A sharp helicity reversion is observed at the position of the Cooper minimum $(\sim 50 \mathrm{eV})$ in Ar. (D) Theoretical simulations confirm the generation of bright, highly chiral CPHHG spectra in the cutoff region.

Finally, we show that the generation of highly elliptical APTs can be achieved without manipulation of either the driving laser field or conditions of the generating medium. By driving the CPHHG process with a noncommensurate, $\omega-0.4 \omega$ field, we generate bright circularly polarized high-harmonics spanning the Cooper minimum in $\operatorname{Ar}$ (Fig. 1C). The presence of the Cooper minimum results in a natural suppression of RCP harmonic orders, yielding highly chiral CPHHG spectra in the high energy cut-off region (Fig. 1C and Fig. 2C). Combined with effective phase-matching in the high-pressure waveguide, we generate a single-helicity CPHHG spectrum, which naturally corresponds to circularly polarized APTs [6]. Moreover, this single-helicity region covers many prominent magneto-optical absorption edges, which should allow for spectroscopies of ultrafast magneto-optical phenomenon.

In summary, we have shown the spectral chirality, and thus the ellipticity of the APTs, in CPHHG can be actively controlled via two straightforward and simple methodologies. As such, highly elliptical APTs can be readily generated and controlled in real-time, thus greatly extending the applicability of these novel light sources to attosecond spectroscopies of chiral dynamics in a variety of condensed matter and molecular systems.

[1] A. D. Bandrauk, J. Guo, K. J. Yuan, "Circularly polarized attosecond pulse generation and applications to ultrafast magnetism," J. Opt. In Press. https://doi.org/10.1088/2040-8986/aa9673

[2] K. M. Dorney, et al., "Helicity-selective enhancement and polarization control of attosecond high harmonic waveforms driven by bichromatic circularly polarized laser fields," Phys. Rev. Lett. 119, 063201 (2017).

[3] N. Zhavoronkov, M. Ivanov, "Extended ellipticity control for attosecond pulses by high harmonic generation," Opt. Lett.42, 4720 (2017).

[4] D. Baykusheva, et al., "Signatures of Electronic Structure in Bicircular High-Harmonic Spectroscopy," Phys. Rev. Lett. 119, 203201 (2017).

[5] C. Hernández-García, et al., "High-order harmonic propagation in gases within the discrete dipole approximation," Phys. Rev. A. 82, 033432 (2010).

[6] D. B. Milošević. "Low-frequency approximation for high-order harmonic generation by bicircular laser field," Phys. Rev. A. In Review. 